\title{
Estudio de la relación topográfica del tercer molar inferior con el conducto mandibular: frecuencia y complicaciones
}

\author{
María M. González, Ph.D. , Gabriela G. Bessone*, Ph.D., \\ Estefanía R. Fernández, Od. ${ }_{3}$, Carlos A. Rosales, Od. \\ 1 Departamento de Clínicas Integradas, Área de Formación Profesional \\ ${ }_{2}$ Departamento de Ciencias Básicas, Área de Formación Básica \\ Odontólogo., becarios de investigación \\ Facultad de Odontología, Universidad Nacional del Nordeste, Corrientes, Argentina
}

Recibido: 25 de febrero del 2016 Aprobado: 28 de septiembre del 2016

*Autor de correspondencia: Gabriela G. Bessone. Profesora Adjunta Introducción a la Odontología, founne, av. Libertad 5450, Campus Deodoro Roca, Corrientes, Argentina. Tel: +54 379 4350079. Correo electrónico: gbessone@odn.unne.edu.ar

Cómo citar este artículo: González MM, Bessone GG, Fernández ER, Rosales CA. Estudio de la relación topográfica del tercer molar inferior con el conducto mandibular: frecuencia y complicaciones. Rev Nac Odontol. 2017;13(24):47-54. doi: http:// dx.doi.org/10.16925/od.v12i24.1666

Resumen. Introducción: el conocimiento de la anatomía de la región mandibular es fundamental para la realización de intervenciones quirúrgicas, debido a la relación de vecindad existente entre las estructuras dentarias y óseas. Objetivos: determinar el trayecto del conducto mandibular como conducto verdadero hasta el agujero mentoniano en maxilares secos, establecer la frecuencia de relación del tercer inferior molar con el conducto, así como registrar las complicaciones que se presentaron durante la erupción del tercer molar inferior, antes y después del tratamiento quirúrgico, según sexo y edad. Métodos: se utilizaron 50 huesos mandibulares secos, silicona con agregado de sulfato de bario, alambre de ortodoncia, compás de punta seca, regla milimetrada y películas radiográficas. Se practicaron cortes frontales y sagitales con el fin de obtener las distancias base, reborde alveolar y diente-conducto. Se emplearon el método porcentual y la prueba de chi-cuadrado $(\mathrm{x} \sim)$ para la cuantificación y la validación de los datos, con una significación estadística de $\mathrm{p}<0,05$. Resultados: se determinó que el conducto dentario inferior es verdadero en el $80 \%$ de los casos. En cortes frontales, el conducto se ubicó más cercano al reborde alveolar. En cortes sagitales la distancia diente-conducto fue íntima. Los datos arrojaron mayor frecuencia de complicaciones en pacientes del sexo femenino, en edades comprendidas entre los 17 y los 25 años, y con posición mesioangular y retenida de la pieza dentaria. Las complicaciones más evidentes fueron trismus, pericoronaritis, dolores de tipo neurálgico y otitis.

Palabras claves: anatomía, mandíbula, nervio mandibular, tercer molar. 


\title{
Study of the topographic relationship between the lower third molar with the mandibular canal: Frequency and complications
}

\begin{abstract}
Introduction: Knowledge of the anatomy of the mandibular region is essential to the performance of surgical interventions due to the existing vicinity relationship between dental and bone structures. Objectives: To determine the path of the mandibular canal as a true canal to the mental foramen in dry mandibles, establish frequency of the relation between the third lower molar and the canal, and record the complications occurring during the eruption of the lower third molar, before and after surgical treatment, by sex and age. Methods: 50 dry mandibular bones, silicone with barium sulfate aggregate, orthodontic wire, dry point compass, millimeter ruler and radiographic films were used. Frontal and sagittal cuts were performed in order to obtain baseline, alveolar ridge and tooth-canal distances. The percentage method and the chi-square test $(\mathrm{x} \sim)$ were used for quantification and validation of the data, with a statistical significance of $\mathrm{p}<0.05$. Results: It was determined that the mandibular canal is true in $80 \%$ of the cases. In frontal cuts, the canal was located closer to the alveolar ridge. In sagittal sections, the tooth-canal distance was intimate. The data showed a higher frequency of complications in female patients, aged between 17 and 25, and with a mesioangular and retained position of the tooth. The most evident complications were trismus, pericoronitis, neuralgic pain and otitis.
\end{abstract}

Keywords: anatomy, mandible, mandibular nerve, third molar.

\section{Estudo da relação topográfica do terceiro molar inferior com o canal mandibular: frequência e complicações}

Resumo. Introdução: o conhecimento da anatomia da região mandibular é fundamental para a realização de intervenções cirúrgicas, devido à relação de proximidade existente entre as estruturas dentárias e ósseas. Objetivos: determinar o trajeto do canal mandibular como canal verdadeiro até o buraco mentoniano em maxilares secos, estabelecer a frequência de relação do terceiro molar inferior com o canal, bem como registrar as complicações que se apresentaram durante a erupção do terceiro molar inferior, antes e depois do tratamento cirúrgico, de acordo com o sexo e a idade. Métodos: foram utilizados 50 ossos mandibulares secos, silicone impregnado de sulfato de bário, arame ortodôntico, compasso de ponta seca, régua milimetrada e películas radiográficas. Foram realizados cortes frontais e sagitais com o objetivo de obter as distâncias base, borda alveolar e dente-canal. Foram empregados o método percentual e o teste de qui-quadrado (x ) para a quantificação e a validação dos dados, com uma significação estatística de $\mathrm{p}<0,05$. Resultados: determinou-se que o canal dentário inferior é verdadeiro em $80 \%$ dos casos. Em cortes frontais, o canal foi posicionado mais próximo à borda alveolar. Em cortes sagitais a distância dente-canal foi íntima. Os dados indicaram maior frequência de complicações em pacientes do sexo feminino, em idades compreendidas entre os 17 e os 25 anos e com posição mesioangular e retenção da peça dentária. As complicações mais evidentes foram trismo, pericoronarite, dores de tipo neurálgico y otite.

Palavras-chave: anatomia, mandíbula, nervo mandibular, terceiro molar. 


\section{Introducción}

El hueso mandibular del niño y del adulto está recorrido en su interior por un conducto denominado "dentario inferior" o mandibular; en él se aloja el paquete vásculo-nervioso del mismo nombre, destinado a la irrigación e inervación de la mandíbula.

Embriológicamente, hacia la cuarta semana de desarrollo se produce la formación de los arcos branquiales y, fundamentalmente, del primer arco branquial, el cual tendrá vital importancia en la morfogénesis facial [1].

A nivel de la mandíbula, el cartílago de Meckel sirve de estructura primitiva de sostén, luego reemplazada por el tejido óseo a través de un tipo de osificación llamada "yuxtaparacondral". El esbozo de mandíbula se presenta como un canal abierto hacia arriba, en el que se encuentra de abajo hacia arriba la vena de Serres, el paquete vásculo-nervioso y los folículos dentarios en desarrollo. Tanto la lámina interna, como la externa, crecen hacia delante llegando a ocupar la sínfisis a la octava semana, momento en que esta queda incluida en la mandíbula ósea. El cuerpo mandibular termina en el sitio donde el paquete sufre una brusca desviación hacia arriba.

En el tercer mes aparecen en el tejido conjuntivo mandibular otros cartílagos, los cuales no derivan del cartílago de Meckel, son los cartílagos secundarios condíleo y sinfisiario. Del primero derivan el cóndilo de la mandíbula, el menisco de la articulación témporo-mandibular y la superficie articular del temporal, mientras que el sinfisiario participa en el crecimiento de esta región mandibular en los planos frontal, sagital y horizontal [2].

La formación de las apófisis alveolares se produce a medida que aparecen los tabiques verticales que separan los alvéolos y más tarde se forma un tabique horizontal que separa a los folículos dentarios del paquete vásculo-nervioso. La estructura del tejido óseo esponjoso de esta zona sufrirá una serie de modificaciones, de acuerdo con la actividad funcional de las piezas dentarias.

El conducto mandibular del niño y del adulto reproducen el canal primitivo del feto, transformado en conducto por la sucesiva formación de gérmenes de la dentición temporaria y permanente, los que generan, a su vez, el desarrollo de sus respectivos alvéolos, factores que en definitiva completan su pared superior.
En el recién nacido, la presencia de un conducto independiente del dentario inferior, denominado "conducto de Serres", es constante en el 100\% de los casos. Su orificio de entrada está situado por detrás y abajo del conducto mandibular, y su orificio de salida se localiza en la cara externa del maxilar, entre la sínfisis y el agujero mentoniano. Corre describiendo una suave curva de concavidad superior hasta su orificio terminal y desaparece normalmente entre los nueve y 10 años de edad. En caso de que persista, se puede considerar la existencia de una alteración en el proceso de crecimiento y osificación del hueso mandibular.

Anatómicamente, el conducto dentario inferior se inicia en la cara interna de la rama ascendente a nivel de la espina de Spix o língula mandibular. Desde su inicio, a nivel del orificio superior, desciende en el interior del hueso y se dirige hacia abajo, adentro y afuera, desembocando en la cara externa a la altura de los premolares, en el agujero mentoniano [3].

El conducto en su recorrido describe una curva de concavidad anterior, se divide en dos segmentos (uno posterior y otro anterior): horizontal hasta el agujero mentoniano donde se bifurca en un conducto mentoniano y otro conducto incisivo; oblicuo hacia abajo y adelante en la rama montante, se hace horizontal en la parte posterior del cuerpo y casi oblicuo arriba, adelante y afuera a partir del ápice del segundo premolar y pasa a 5 milímetros del ápice del tercer molar inferior.

En su interior el hueso mandibular está constituido por una masa central esponjosa densa, de manera que puede denominarse "tejido esponjoso" al que rodea al conducto dentario inferior [4]. Por arriba, este conducto comienza en la cara interna de la rama, por detrás de la espina de Spix; desde este punto se dirige oblicuamente hacia abajo y adelante. A la altura de los premolares se divide en dos ramas: interna y externa. Este conducto posee un número de conductos secundarios verticales que desembocan en cada alvéolo [5].

El conducto dentario inferior se encuentra a 8 o 9 milímetros por encima del borde inferior de la mandíbula. En su trayecto pasa algunos milímetros por debajo de los ápices dentarios, aunque pueden existir casos en los que se presenta lateral, lingual o esté ubicado bucalmente, con respecto a las raíces dentarias. Con relación al tercer molar, normalmente pasa a 5 milímetros de su ápice y en casos de inclusión puede ubicarse entre sus dos raíces [6]. 
En cortes frontales, tiene un aspecto de círculo u óvalo de 2 a 3 milímetros de diámetro. En estado fresco está ocupado por el nervio dentario inferior, la arteria del mismo nombre y venas satélites.

Oliver describe dos tipos anatómicos de conductos, uno verdadero con paredes gruesas, de color blanco nacarado, que se presenta en el $60 \%$ de los casos, y uno mal individualizado en el tejido esponjoso, que aparece en el $40 \%$ restante [7].

El conducto dentario inferior posee una cortical ósea propia, nítidamente radiopaca, imagen radiográfica que contrasta con el tejido óseo que lo rodea y con la imagen radiolúcida de su trayecto, la cual consiste en una pequeña laminilla compacta que corre por el tejido esponjoso del hueso [8].

Topográficamente, la relación entre las piezas dentarias y el conducto puede ser de tres tipos: 1) el más frecuente es aquel cuya distancia entre conducto y ápices, disminuye lentamente, de adelante hacia atrás, hasta llegar al tercer molar, donde la distancia se hace íntima al estar separado por una delgada capa de tejido esponjoso; 2) los ápices se encuentran a una distancia considerable del conducto, y 3) todos los dientes en relación con el conducto dentario llegan hasta él [9].

Por su parte, las relaciones del tercer molar con el conducto dentario se clasifican en cuatro tipos: a) en el más frecuente, el conducto corre muy vecino a los ápices dentarios de los molares, encontrándose una exigua cantidad de tejido óseo compacto separándolos; b) el menos frecuente, es aquel en el que existe un amplio espacio entre ápices y conducto; c) es raro el caso en que los ápices se introducen en la luz del conducto; d) el conducto se ubica hacia bucal o lingual de los ápices.

Los valores promedios referidos al espesor del hueso que separa los ápices dentarios del conducto mandibular son: tercer molar, 2 a $6 \mathrm{~mm}$; segundo molar, 5 a $7 \mathrm{~mm}$; primer molar, 6 a $9 \mathrm{~mm}$; segundo premolar, $8 \mathrm{~mm}$ [10].

Cabe consignar que, en los casos en los que existe una marcada proximidad entre la raíz del tercer molar y el conducto mandibular, pueden producirse alteraciones como inflamación del ligamento periodontal, dolor neurálgico, pos extracción, o bien producir durante el acto operatorio un aplastamiento de los elementos vásculo-nerviosos con consecuencias tales como neuritis, anestesias y/o parestesias [11, 12].

En cortes horizontales, se puede apreciar que el conducto se encuentra muy cercano a la tabla interna, a la altura del primer y segundo molar, siendo la distancia mínima de un milímetro. El punto declive se ubica a un centímetro por encima del borde inferior de la mandíbula, a la altura de ambos molares.

Dado lo anterior, los objetivos de la investigación fueron determinar el trayecto del conducto dentario inferior como conducto verdadero, verificar la relación existente con el tercer molar, y evaluar las complicaciones más frecuentes que se presentan durante la erupción del tercer molar, antes y después de un tratamiento quirúrgico, teniendo como variables el sexo y la edad $[13,14]$.

\section{Materiales y métodos}

El trabajo se llevó a cabo en la cátedra Introducción a la Odontología, con la colaboración de las cátedras de Clínica Estomatológica, Cirugía III, Cirugía y Traumatología Buco máxilofacial, y la de Servicio de Diagnóstico por Imágenes, de la Facultad de Odontología de la Universidad Nacional del Nordeste.

En el laboratorio se trabajó con 50 huesos mandibulares secos, silicona con agregado de sulfato de bario, alambre de ortodoncia, compás de punta seca, regla milimetrada y películas radiográficas periapicales, oclusales y panorámicas.

En clínica se atendieron 100 pacientes de ambos sexos, cuyas edades oscilaron entre 15 y 30 años, incluyendo a aquellos que presentaron accidentes y complicaciones de los terceros molares inferiores durante su erupción, así como antes y después de la cirugía del tercer molar.

Se excluyeron del estudio a mujeres embarazadas, pacientes con disfunciones témporo-mandibulares, diabéticos y pacientes con discrasias sanguíneas.

Se realizó el ordenamiento de los huesos mandibulares secos, tomando como referencia para su codificación, la angulación obtenida entre las líneas trazadas a nivel de la mitad de la escotadura sigmoidea, hasta el ángulo de la mandíbula en sentido vertical, y desde la sínfisis mentoniana hasta la mitad del borde posterior de la mandíbula en sentido horizontal.

Luego se procedió a colocar dentro del conducto el material siliconado, logrando marcar el trayecto del mismo desde su origen y hasta el conducto mentoniano. Una vez realizado este 
procedimiento, se comenzó la toma de radiografías oclusales de los huesos secos a fin de estudiar radiográficamente el trayecto.

Posteriormente, se realizaron cortes frontales y sagitales con el fin de tomar las distancias entre base-conducto, conducto-reborde alveolar, alvéolo del $3 \mathrm{M}$ y techo del conducto mandibular. Para ello se utilizó el compás, y luego esa medida se trasladó a una regla milimetrada para la obtención de los datos y el registro correspondiente en una planilla confeccionada para tal fin.

Clínicamente, se evalúo a los pacientes durante el período 2013-2014, quienes concurrieron espontáneamente a las clínicas de la facultad, luego de obtener su consentimiento informado para integrar la muestra, y después de una exhaustiva anamnesis y examen clínico, se confeccionaron las historias clínicas y fichas odontológicas. Mediante la toma de radiografías, se obtuvo el diagnóstico presuntivo, conformándose dos grupos de trabajo: pacientes con complicaciones a nivel del tercer molar inferior antes del tratamiento quirúrgico y pacientes que presentaron complicaciones después del tratamiento exodóntico.

Los datos obtenidos fueron ordenados y analizados teniendo en cuenta edad, sexo y los tipos de complicaciones, así como al analizar la relación de vecindad a través de radiografías, con base en los siguientes parámetros: Íntima: hasta $1 \mathrm{~mm}$; Cercana: de 1,1 a $2 \mathrm{~mm}$; Lejana: 2,1 o más mm.

Asimismo, se evaluaron las complicaciones más frecuentes tales como dolores neurálgicos, molestias leves, pulpitis, trismus, asimetrías faciales, parálisis, parestesias, y se establecieron dos categorías: 1) presencia de complicaciones y 2) ausencia de complicaciones.

A fin de cuantificar los resultados, se realizó un análisis estadístico de tipo descriptivo, y para la validación se utilizó el chi-cuadrado $\left(\mathrm{X}^{2}\right)$, con una significación estadística de $\mathrm{p}<0,05$.

\section{Resultados}

De la marcación del trayecto del conducto dentario inferior con silicona adicionada con sulfato de bario y alambre de ortodoncia, mediante los cortes sagitales de los maxilares secos, el $80 \%$ de los mismos presentaron conductos con paredes bien delimitadas, techo y piso marcados durante todo su recorrido, hasta el conducto mentoniano. El 20\% presentó conductos con paredes bien delimitadas $y$, en parte, pasaje o galería excavada en el tejido óseo esponjoso.

En cortes frontales, se observó que todos los conductos adoptaban una forma redondeada u ovalada, con un diámetro mayor en sentido vertical y un color blanco nacarado (véanse las figuras 1 y 2).

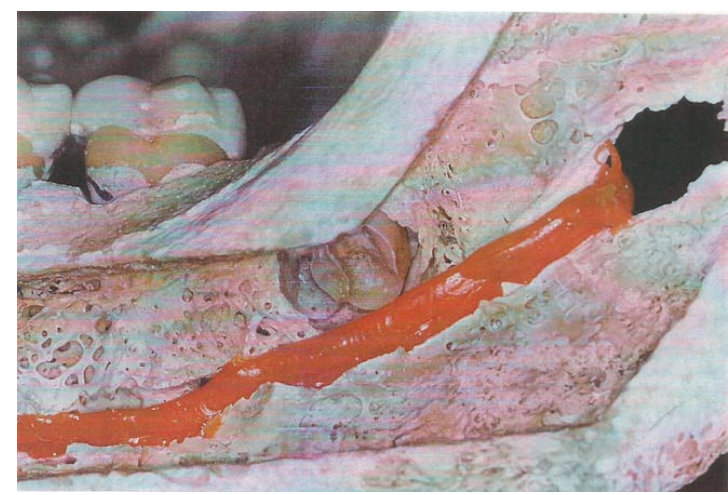

Figura 1. Recorrido del conducto mandibular con silicona conteniendo sulfato de bario y alambre en corte sagital Fuente: Cátedra Clínica Estomatológica

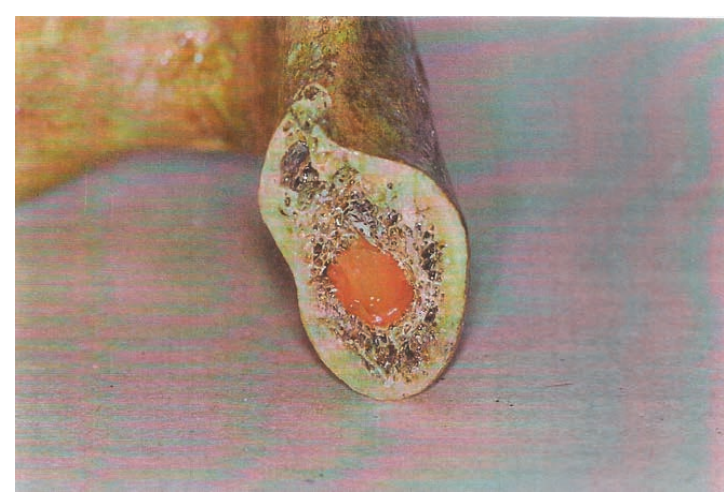

Figura 2. Corte frontal del conducto mandibular con silicona Fuente: Cátedra Clínica Estomatológica

Con respecto a las distancias, se observó que en relación base-conducto, la más frecuente fue de 1,6 a $2 \mathrm{~mm}$, en el $54 \%$ de los casos. Con respecto a la distancia reborde alveolar-conducto, fue de 1,5 a $2,5 \mathrm{~mm}$, en el $48 \%$, mientras que la distancia diente-conducto, fue de 1 a 2,5, en el $64 \%$ de los casos (véanse las Tablas 1, 2 y 3 ). 
Tabla 1. Distancia base-conducto obtenidos en cortes frontales de mandíbulas secas

\begin{tabular}{|l|c|c|}
\hline Distancia en mm & $\begin{array}{c}\text { Frecuencia } \\
\text { absoluta (Fa) }\end{array}$ & $\begin{array}{c}\text { Frecuencia } \\
\text { relativa (Fr) }\end{array}$ \\
\hline $1-1,5 \mathrm{~mm}$ & 20 & 40 \\
\hline $1,6-2 \mathrm{~mm}$ & 27 & 54 \\
\hline Más de $2 \mathrm{~mm}$ & 03 & 06 \\
\hline Total & 50 & 100 \\
\hline
\end{tabular}

Fuente: Cátedra Clínica Estomatológica

Tabla 2. Distancia reborde alveolar-conducto obtenida en cortes frontales de mandíbulas secas

\begin{tabular}{|l|c|c|}
\hline Distancia en mm & $\begin{array}{c}\text { Frecuencia } \\
\text { absoluta (Fa) }\end{array}$ & $\begin{array}{c}\text { Frecuencia } \\
\text { relativa (Fr) }\end{array}$ \\
\hline $1,5-2,5 \mathrm{~mm}$ & 24 & 48 \\
\hline $2,6-3,5 \mathrm{~mm}$ & 14 & 28 \\
\hline $3,6-5 \mathrm{~mm}$ & 12 & 24 \\
\hline Total & 50 & 100 \\
\hline
\end{tabular}

Fuente: Cátedra Clínica Estomatológica

Tabla 3. Distancia tercer molar-conducto, obtenida en cortes sagitales de mandíbulas secas

\begin{tabular}{|l|c|c|}
\hline \multicolumn{1}{|c|}{ Distancia en $\mathbf{m m}$} & $\begin{array}{c}\text { Frecuencia } \\
\text { absoluta }(\mathrm{Fa})\end{array}$ & $\begin{array}{c}\text { Frecuencia } \\
\text { relativa (Fr) }\end{array}$ \\
\hline $1,5-2,5 \mathrm{~mm}$ & 32 & 64 \\
\hline $2,6-4 \mathrm{~mm}$ & 12 & 24 \\
\hline $4-6 \mathrm{~mm}$ & 06 & 12 \\
\hline Total & 50 & 100 \\
\hline
\end{tabular}

Fuente: Cátedra Clínica Estomatológica
La distribución por edades en los casos clínicos tratados, mostraron que el grupo etáreo más afectado (en un 85\%) fue el de los 17 a 25 años y, en cuanto al sexo, el predominio fue para el femenino, en un $63 \%$.

Se registraron diferencias significativas entre sexo, complicaciones y distancia diente-conducto. Mientras el sexo femenino fue afectado con 32 casos y la distancia fue íntima, el sexo masculino solo presentó 18 casos y mayores distancias diente-conducto.

De las complicaciones a distancia, las más observadas fueron los dolores de tipo neurálgico en el $82 \%$ de los casos y otitis en el $17 \%$.

Las complicaciones posquirúrgicas fueron el trismos (10\%), seguido de las parestesias (3\%), siendo el sexo femenino el más afectado (10\%).

\section{Discusión}

El conducto dentario inferior estudiado se encuentra localizado en la cara interna del maxilar inferior, desde su inicio a la altura de la rama ascendente a nivel del orificio del mismo nombre, protegido por la espina de Spix o lígula mandibular. Este orificio es la puerta de entrada del conducto y continúa en el interior del hueso, terminando a la altura del primer premolar, o bien en el espacio comprendido entre ambos premolares en el conducto mentoniano o interno. Luego del conducto madre, se desprende una prolongación hacia delante denominada "conducto incisivo" o "externo", en coincidencia con los autores analizados $[16,18,21,24]$.

Tabla 4. Estudio radiográfico de las complicaciones locales ocasionadas por el tercer molar inferior en relación con su erupción

\begin{tabular}{|c|c|c|c|c|c|c|}
\hline Posición & & & & & & \\
\hline Complicaciones & SÍ & $\mathrm{NO}$ & SÍ & $\mathrm{NO}$ & SÍ & NO \\
\hline Г & 03 & 33 & 10 & 26 & 20 & 16 \\
\hline & $8,3 \%$ & $91,7 \%$ & $27,8 \%$ & $72,2 \%$ & $55,6 \%$ & $44,4 \%$ \\
\hline 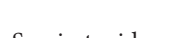 & 10 & 12 & 07 & 35 & 16 & 26 \\
\hline & $45,5 \%$ & $54,5 \%$ & $16,7 \%$ & $83,3 \%$ & $38,1 \%$ & $61,9 \%$ \\
\hline & 23 & 19 & 02 & 20 & 12 & 10 \\
\hline & $54,8 \%$ & $45,2 \%$ & $9,1 \%$ & $90 \%$ & $54,5 \%$ & $45,5 \%$ \\
\hline Totrot & 36 & 64 & 19 & 81 & 48 & 52 \\
\hline & $36 \%$ & $64 \%$ & $19 \%$ & $81 \%$ & $48 \%$ & $52 \%$ \\
\hline
\end{tabular}

Fuente: Cátedra Clínica Estomatológica 
La observación macroscópica de cortes sagitales practicados en maxilares secos ha demostrado que, en el $80 \%$ de los casos, el conducto se presentó como único y verdadero en su recorrido hasta el conducto mentoniano y solo el $20 \%$ de los casos presentaron paredes delimitadas con trayecto poco nítido, coincidiendo con Velayos y Díaz-Santana [5].

Respecto a la morfología del canal mandibular, Olivier, citado por Anderson et al., lo describió como oval, piriforme y circular, al realizar cortes sagitales, con un diámetro entre 2,0 y $2,4 \mathrm{~mm}$. Con relación a la dirección, este mismo autor describió un curso cóncavo con un segmento posterior descendente y uno anterior que se dirige hacia adelante ascendiendo hasta el agujero mentoniano. Encontró que el canal está $6 \mathrm{~mm}$ por debajo de la raíz del tercer molar, y 7,8 y $9 \mathrm{~mm}$ de las raíces del segundo molar, primer molar y segundo premolar, respectivamente, al igual que los resultados obtenidos en este trabajo $[19,20]$.

Con respecto a las distancias mínimas y máximas halladas entre las piezas dentarias y el conducto, fueron de 1 a $6 \mathrm{~mm}$, siendo el tercer molar inferior, la pieza dentaria que con mayor frecuencia se relaciona con dicho elemento anatómico, coincidiendo con Dielafeu y Herpin, citados por Figún y Garino, quienes realizaron investigaciones en cuanto a las distancias y la relación diente-conducto, en las cuales encontraron como distancias mínimas y máximas un rango entre 2 a $6 \mathrm{~mm}$. Estos valores confirman los enunciados por Oliver $[2,14$, $15,17]$. Las medidas observadas con mayor frecuencia fueron de 1 a $2,5 \mathrm{~mm}$, en un $64 \%$.

La ubicación del conducto en cortes frontales es más cercana al reborde alveolar que al reborde basal, siendo la distancia mínima de 1,5 mm, mientras que la distancia diente-conducto fue de 1 a 2,5 $\mathrm{mm}$. Esto se explicaría porque el conducto dentario inferior se desarrolla embriológicamente mucho antes que el germen del tercer molar, de manera que las raíces dentarias, cuando adquieren normal desarrollo, pueden entrar en contacto con la pared ósea del conducto, rodearlo total o parcialmente. Wang, Shih, Liu y Kuo [22], Phillips, Weller y Kulild [23] y Shankland [24] coinciden que el conducto dentario inferior se relaciona íntimamente con los alvéolos de molares y premolares, en consecuencia con el tercer molar inferior, siendo necesario extremar los cuidados durante la intervención quirúrgica a fin de evitar accidentes y complicaciones [2].
En los casos clínicos evaluados, la distribución por edades de los pacientes demostró fuerte asimetría, siendo el grupo etáreo más significativo el de 17 a 25 años, con una media de 21,8. El sexo más afectado fue el femenino coincidiendo con Bozzatello [21], quien describe el ligero predominio del sexo femenino en la presentación de complicaciones durante la erupción del tercer molar inferior.

Con respecto a las diferencias encontradas en relación sexo y distancia diente-conducto, las mujeres se encontraron en la categoría de menos de 1 milímetro. Esto se debería al tamaño que adoptan los maxilares inferiores en las mujeres, quienes tienen mayor tendencia a la retención de los terceros molares inferiores, siendo los factores que afectan el normal desarrollo y la erupción: la falta de espacio en el sector posterior, factores concomitantes con el ángulo goníaco, el ángulo inciso maxilar y otros factores tales como retardo y dirección en el crecimiento, dirección de la erupción, incidencia de la línea oblicua externa y músculo buccinador.

Se hallaron diferencias entre la posición de los terceros molares inferiores durante su erupción y las complicaciones que presentaron, siendo las piezas retenidas en posición mesioangular las más frecuentes [21]. Esto indicaría que la actuación mecánica de la pieza dentaria impactada podría ocasionar trastornos durante su ubicación en el maxilar, ocasionando alteraciones de posición en los dientes vecinos, o bien generando apiñamiento a nivel de incisivos.

También pueden observarse trastornos en la integridad anatómica del tercer molar cuando este ejerce presiones sobre el diente vecino, causando alteraciones en los tejidos dentarios. De allí la importancia de precisar la posición mediante tomas radiográficas, las cuales constituyen un método auxiliar para corroborar el diagnóstico presuntivo y planificar el tratamiento.

Las complicaciones locales observadas antes del tratamiento fueron trismos y pericoronaritis.

Las complicaciones a distancia observadas fueron los dolores tipo neurálgico y dolores en la zona del trago, los cuales podrían vincularse a dolores irradiados a zonas vecinas.

\section{Conclusiones}

El conducto dentario inferior se presentó verdadero y único en su trayecto hasta el conducto 
mentoniano en un $80 \%$, presentando durante todo su recorrido paredes bien definidas y delimitadas, en relación de proximidad con el alvéolo del tercer molar.

El grupo etáreo más afectado en un $85 \%$ fue el de 17 a 25 años, y en cuanto al sexo, el predominio fue para el femenino en un 63\%. Considerar estas variables en la atención odontológica es fundamental debido a la íntima relación del tercer molar con el conducto mandibular.

La complicación hallada con mayor frecuencia antes de la exodoncia fue la pericoronaritis, y después de la cirugía, el trismus.

El conocimiento de la anatomía del canal mandibular y las relaciones de vecindad permite trabajar de manera segura y eficaz, evitando los riesgos que implican las complicaciones nerviosas asociadas al daño del nervio dentario inferior.

\section{Referencias}

[1] Ash M, Stanley N. Anatomía, fisiología y oclusión dental. 8a ed. España: Elsevier; 2003.

[2] Figun M, Garino R. Anatomía odontológica funcional y aplicada. $2^{\text {a }}$. ed. Buenos Aires: El Ateneo; 2002.

[3] Diamond M. Anatomía dental. $2^{\text {a }}$ ed. México: Interamericana; 2010.

[4] Kraus B, Jordan R. Abrams L. Anatomía Dental y Oclusión. $1^{\text {a }}$ ed. México: Interamericana; 1972.

[5] Velayos J, Diaz-Santana H. Anatomía de la Cabeza con enfoque Odontoestomatológico. Madrid: Médica Panamericana; 1994.

[6] Visvisián C, Gani-Artola O, Abdo O. Ulfohn-Pave, R. Cambios producidos por la edad en la forma de los conductos radiculares del primer molar superior. Rev Asoc Odontol Argent. 1997; 85(2):129-34.

[7] Latarjet M, Ruiz-Liard A. Atlas de Anatomía. 2a . ed. Buenos Aires: Panamericana; 2004.

[8] Rouviere H. Anatomía Humana, Descriptiva, Topográfica y Funcional. $8^{\mathrm{a}}$ ed. Madrid: Bailly Bailliere SA; 1970.

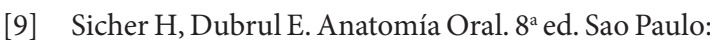
Artes Médicas; 1991.

[10] Carbo-Ayala J. Anatomía dental y de la oclusión. La Habana: Ciencias Médicas; 2009.

[11] Brand R, Isselhard D. Anatomía de la Estructuras Orofaciales. España: Harcourt Brace; 1999.

[12] Diamond M. Anatomía Dental. México: Interamericana; 1992.

[13] Aprile H, Figún M, Garino RR. Anatomía Odontológica, Orocervical. $5^{\mathrm{a}}$ ed. Argentina: El Ateneo; 1984.

[14] Sobotta J. Atlas de Anatomía Humana. 19ª ed. Madrid: Panamericana; 1991.

[15] Girard K. Considerations in the management of damage to the mandibular nerve J. Am. Dent. Assoc. 1979 ; 98:65-7.

[16] Tortora G, Grabowsky SR. Principios de Anatomía y Fisiología. $7^{\text {a }}$ ed. España: Mosby Dogma Libros; 1996.

[17] Manns A. Sistema Estomatognático. Chile: Sociedad Gráfica de Almagro Ltda; 1988.

[18] Ash MS. Anatomía dental fisiología y oclusión. 7 ed. México: Interamericana; 1994.

[19] Gravosky S. Principios de Anatomía y Fisiología. $7^{\text {a }}$ ed. España: Mosby Dogma Libros; 1996.

[20] Anderson L, Kosinski T, Mentag PJ. A review of the intraosseous course of the nerves of the mandible. J Oral Implantol. 1991;17(4):394-403.

[21] Bozzatello JR. Estudio estadístico del tercer molar inferior retenido, en la ciudad de Córdoba (Argentina). Tesis de Doctorado. Rev Fac Odontol Univ Nac (Córdoba). 2002. Disponible en: http://hdl.handle. net/11086/1733

[22] Wang TM, Shih C, Liu JC, Kuo KJ. A clinical and anatomical study of the location of the mental foramen in adult chinese mandibles. Acta Anat. 1986;126(27):29-33.

[23] Phillips JL, Weller RN, Kulild JC. The mental foramen: Size, orientation, and positional relationship to the mandibular second premolar. J Endod. 1990;16(28):221-223.

[24] Shankland WE. The position of the mental foramen in asían indians. J Oral Implanto. 1994;20:118-123. 